\title{
Abstracts
}

\section{Urology Session 2}

Urinary Antimicrobial Peptide Expression is a Marker of a Neurogenic Bladder Regardless of Infection or Colonization

Preece, Janae ${ }^{2}$, Gupta, Sudipti ${ }^{1}$, Watson, Joshua ${ }^{1}$, Baxter, Cheryl ${ }^{1}$, Riesenberg, Stephanie ${ }^{1}$, Haynes, Andria $^{1}$, Becknell, Brian ${ }^{1}$, Ching, Christina ${ }^{1}$

${ }^{1}$ Nationwide Children's Hospital

${ }^{2}$ Children's Hospital of Michigan

Background Bacteriuria is common in the myelomeningocele (MMC) population. Distinguishing between bacterial colonization versus infection can be difficult and result in antibiotic overuse leading to resistant bacteria. We evaluated urinary antimicrobial peptides (AMPs) as potential biomarkers to differentiate between MMC patients with colonization versus true infection.

Methods Urine was collected by void or catheterization from patients with a history of MMC or tethered cord at time of renal ultrasound or urodynamics according to an IRB-approved protocol. Patients with augmentation cystoplasty were excluded. Control urine was obtained from patients without spinal abnormality or urinary obstruction/infection. Samples were classified as infected, colonized, or sterile. AMP levels were measured by ELISA and normalized to urine creatinine. A p-value of $<0.05$ was considered significant.

Results We evaluated $41 \mathrm{MMC}$ and 17 control samples. There was no difference in age between groups (mean age 11.7 vs. 12.9 years; $p=0.50$ ). Of the MMC samples, 11 were sterile, 22 colonized, and 8 infected. HIP/PAP, BD-1, Def-5, and LL-37 levels were significantly higher in sterile MMC samples compared to controls $(p<0.0001, p=0.001, p=0.009, p=$ 0.003 , respectively). HIP/PAP and BD-1 levels were overall higher in the urine of MMC patients with UTI versus colonization, but did not reach statistical significance (HIP/PAP: mean 0.31 vs. $0.27 \mathrm{ng} / \mathrm{mg}, p=0.40$;
BD-1: mean 517.7 vs. $361.8 \mathrm{ng} / \mathrm{mg}, p=0.75$, respectively).

Conclusions Sterile urine from MMC neurogenic bladders have significantly elevated urinary AMP levels compared to non-neurogenic controls and may suggest a broader role for AMPs in this patient population. Urinary AMPs may aid in differentiating true infection from colonization in neurogenic patients however larger studies are needed.

\section{Factors Contributing to Stone Formation in Myelomeningocele in those with and without Bladder Augmentation}

Dangle, Pankaj P. ${ }^{2}$, Atchley, Travis ${ }^{1}$, Hopson, Betsy D. ${ }^{2}$, Graham, Anna ${ }^{2}$, Arynchyna, Anastasia ${ }^{2}$, Rocque, Brandon G. ${ }^{2}$, Joseph, David B. ${ }^{2}$, Wilson, Tracey S. ${ }^{1}$

${ }^{1}$ University of Alabama School of Medicine

${ }^{2}$ UAB-Children's of Alabama

Background Bladder and renal stones occur after bladder augmentation at a reported rate of $52 \%$, with $31 \%$ of bladder and $60 \%$ of renal stones being noninfectious. The incidence and composition of stones in patients who do not undergo urinary reconstruction is unclear. We sought to determine the incidence and location of urolithiasis in patients with myelomeningocele (MMC) with and without augmentation and factors associated with stone formation.

Methods We performed a retrospective analysis of all patients with MMC in the Children's Of Alabama SB registry with at least one documented stone removal procedure. We performed univariate analysis on our cohorts. We collected stone analysis data, if available. Patients were excluded if they did not have a diagnosis of MMC or did not have a known stone removal.

Results Of 530 total MMC patients, 83 (15.7\%) had bladder augmentation. 15/83 (18.1\%) had a urinary 
stone. In the non-augmentation group, 24/447 (5.4\%) had a known stone. 2/15 (13.3\%) and 9/24 (37.5\%) individuals had upper tract stones in the augmentation and non-augmentation groups, respectively. Of 9 stone analyses in the non-augmentation group, $66.7 \%$ of stones were infectious. In the augmentation group, a ventriculoperitoneal (VP) shunt was associated with stone formation $(p=0.035)$. In the non-augmentation group, presence of VP shunt, non-community ambulation status, high-level spinal lesions (thoracic, highlumbar, and mid-lumbar), wheelchair use were all associated $(p<0.050)$ with stone formation, though clean intermittent catheterization and antibiotic use were not.

Conclusions Based on our analysis, stone disease is seen in $18 \%$ of patients following bladder augmentation. More importantly, of those without urinary reconstruction, $5.4 \%$ of patients had stone disease, and most $(68 \%)$ are infectious. In patients with no augmentation, non-modifiable risk factors contribute significantly to stone disease.

\section{A Novel Predictor for Stone Free Rates After Per- cutaneous Stone Surgery in Spina Bifida Patients Chaudhry, Rajeev, Theisen, Katherine, Stephany, Heidi, Cannon, Glenn, Schneck, Francis, Ost, Michael Children's Hospital of Pittsburgh}

Background Percutaneous stone surgery can be challenging in Spina Bifida (SB) patients due to their body habitus and severe scoliosis. A safe surgical approach may necessitate acceptance of lower stone-free rates. We evaluate factors that may influence stone free rates after percutaneous stone surgery in SB patients.

Methods We retrospectively evaluated our outcomes following percutaneous nephrolithotomy (PCNL) and percutaneous cystolitholapaxy (PC) in patients with Spina Bifida from 2008 to 2016. Patient charts were reviewed for demographic factors, stone characteristics and operative factors. We calculated individual Cobb angles to quantify scoliosis severity to evaluate outcomes based on anatomic complexity.

Results Twenty-three patients underwent 32 procedures (15 PCNL, 17 PC), and overall stone free rates were $62.5 \%$ after a single procedure. Individual stone free rates for PCNL and PC were 53\% and 71\%, respectively, and complication rates were $33 \%$ and $24 \%$, respectively. There were no statistical differences in age, stone burden, BMI, operative time, EBL between the stone-free and not stone-free groups. There were more complications and longer hospital stays in the group of patients deemed stone-free after a single PCNL (4 vs 1 complication, and 4.4 vs 2.1 days, respectively). There was a trend toward higher Cobb angles in patients requiring multiple PCNLs to achieve stone-free status (43 vs. 24 , respectively, $p=0.058$ ).

Conclusions Percutaneous stone surgery in patients with SB was associated with modest stone-free rates and a high complication rate. The use of Cobb angle can be a novel tool to predict success after stone surgery in SB patients, which has not been previously described. Further prospective studies are warranted.

\section{Bladder Deterioration Following Bladder Outlet Procedure without Enterocystoplasty in Spina Bifida: Need for Extended Follow Up \\ VanderBrink, Brian, Reddy, Pramod, Minevich, Eugene, Jackson, Elizabeth, Noh, Paul, Sheldon, Curtis, DeFoor, W. Robert \\ Cincinnati Children's}

Background Neuropathic sphincteric incontinence can be treated with bladder outlet procedure (BOP). When this procedure was performed we previously reported a $33 \%$ incidence of requiring additional bladder procedures such as BoTox injection or enterocystoplasty to address de novo bladder dynamics with median follow up of 2 years. The purpose of this study was to update that prior report with further follow up to determine if the original incidence had changed.

Methods A retrospective cohort study was performed of patients with myelomeningocele who underwent BOP without enterocystoplasty. Clinical, urodynamic and radiographic variables were compared prior to and after BOP for each patient.

Results Thirty-six patients comprised the study group with mean age of 10 years at time of BOP and median time from BOP of 7 years. Twenty patients (56\%) developed postoperative clinical or radiographic signs of bladder dysfunction confirmed by repeat urodynamics. Fifteen patients underwent BoTox injection. Seven patients underwent enterocystoplasty including 2 patients unresponsive to BoTox. There continued to be no preoperative difference between the two groups in terms of cystometric capacity expressed as percentage of age expected bladder capacity, detrusor leak point pressure, bladder compliance or presence of trabeculation, vesicoureteral reflux or hydronephrosis. There was longer time from surgery in the patients that did not undergo additional surgery (median 8 vs. 5 years 
$[p=0.03])$. Patients undergoing bladder surgery were younger than those who did not at time of BOP (9 vs. 12 years $[p=0.04])$.

Conclusions With further time more patients with isolated BOP developed detrusor changes resulting in increased performance of bladder procedures BoTox injection or enterocystoplasty. Younger age of surgery may be associated with increased need for additional bladder surgery with isolated BOP procedure.

\section{Bladder Reconstruction Rates Are Significantly Different Among National Spina Bifida Patient Registry Centers}

Routh, Jonathan C. ${ }^{1}$, Joseph, David B. ${ }^{2}$, Liu, Tiebin ${ }^{3}$, Schechter, MIchael ${ }^{5}$, Thibadeau, Judy ${ }^{3}$, Wallis, Chad ${ }^{4}$, Ward, Elisabeth ${ }^{3}$, Wiener, John S. ${ }^{1}$

${ }^{1}$ Duke University

${ }^{2}$ University of Alabama at Birmingham

${ }^{3} \mathrm{CDC}$

${ }^{4}$ Primary Children's Hospital

${ }^{5}$ Virginia Commonwealth University

Background We performed an exploratory analysis of data from the National Spina Bifida Patient Registry (NSBPR) to assess variation in the frequency of bladder reconstruction surgeries among NSBPR centers.

Methods We queried the 2009-2014 NSBPR to identify patients who had ever undergone bladder reconstruction surgeries. We evaluated demographic characteristics, SB type, functional level, mobility, education, and NSBPR center to determine whether any of these factors were associated with reconstructive surgery rates. Multivariable logistic regression was used to simultaneously adjust for the impact of these factors.

Results We identified 5,596 patients with SB enrolled in the NSBPR. Of these, 1,135 (20\%) underwent bladder reconstruction (706 augmentation, 383 continent catheterizable channel, 190 bladder outlet procedure). Surgery patients were more likely to be older (16.7 vs. 9.8 years, $p<0.001$ ); female $(22.3$ vs. $18.0 \%, p<$ $0.001)$; white (22\% whites vs. $12-18 \%$ other groups, $p<0.001)$; privately insured (21.4 vs. $19.0 \%, p=$ 0.03 ); and to be treated at a NSBPR center with higher bladder reconstruction surgery rates (range 2.7-37.9\%, $p<0.001)$. After correcting for known confounders, NSBPR center, SB type, mobility, gender and age (all $p<0.0001)$ were the most significant predictors of surgical intervention. Race $(p=0.19)$ and insurance status ( $p=0.11$ ) were not associated with surgical intervention.
Conclusions There is significant variation in rates of bladder reconstruction surgery among NSBPR centers. In addition to clinical factors such as mobility status, lesion type, and lesion level, non-clinical factors such as patient age, gender and treating center are also associated with the likelihood of an individual undergoing bladder reconstruction.

\section{Modified Technique of Triamcinolone Injection for the Treatment of Continent Catheterizable Channel Stomal Stenosis: Optimizing Results and Reducing Cost of Care}

Strine, Andrew, VanderBrink, Brian, DeFoor,

W. Robert, Minevich, Eugene, Noh, Paul, Reddy, Pramod

Cincinnati Children's

Background Continent catheterizable channels (CCCs) using the Mitrofanoff principle are often created during continent reconstruction but may develop stomal stenosis in up to $30 \%$ of patients. We describe our experience with a modified technique of triamcinolone injection for the treatment of stomal stenosis, avoiding the need for a more invasive and costly stomal revision.

Methods We performed a retrospective review of patients who underwent a modified technique of triamcinolone injection $(40 \mathrm{mg} / \mathrm{mL})$ for the treatment of stomal stenosis at our institution. Demographic and clinical data were assessed. A successful outcome was defined as a return to ease with catheterization. We also assessed the cost of care with various techniques for the treatment of stomal stenosis by representing the cost as a percentage of charges for a re-operative Monti ileovesicosotmy.

Results A total of 20 injections were performed in 17 CCCs of 16 patients (14 urinary channels and 3 colon channels). Fourteen underwent the injection under general anesthesia, while two patients with poor sensation underwent an office-based procedure. After median follow up of 12 months, thirteen CCCs (76\%) were successfully treated with a single injection. Three patients required a total of 5 repeat injections at a median of 5.1 months between injections (range, 3.218.4), while only 1 patient required a stomal revision at 34.9 months after the initial injection. There were no predictors of failure after the initial or any injection on univariate analysis. The cost of care was $11.3 \%$ for a stomal revision, $5.8 \%$ for Triamcinolone injection in the operating room, and $0.3 \%$ as an office-based procedure. 
Conclusions Our modified technique of triamcinolone injection was a durable option for the treatment of stomal stenosis in any cutaneous stoma with a success rate of $76 \%$ after a single injection.

\author{
Incontinent Urinary Diversion in Young Adult \\ Spina Bifida Patients \\ McNamara, Erin R., Estrada, Carlos, Sherlock, \\ Rebecca \\ Boston Children's Hospital
}

Background Patients with Spina Bifida (SB) are living well into adulthood and present unique challenges for pediatric and adult urologists. An important issue is how to manage the urinary tract of adults with SB who can no longer manage with clean intermittent catheterization (CIC). We present our experience with this young adult population.

Methods With IRB approval, we completed a retrospective chart review of SB patients who underwent incontinent urinary diversion in the last 3 years. We collected demographics, preoperative testing, operative characteristics and postoperative events for these patients to highlight the indications and results of these procedures.

Results Six patients between 2012 and 2014 underwent incontinent urinary diversion. Indications were worsening renal function and/or incontinence and inability to manage a bladder augment and/or continent catheterizeable stoma.

Patient characteristics: The median age at time of surgery was 28.3 years (range 20.5-33). 5 (83\%) patients had significant comorbidities, including hypertension, chronic kidney disease, severe restrictive lung disease, and/or sleep apnea. Prior to surgery, all patients underwent medical evaluation.

Surgical characteristics: Median operative time was 313 minutes (range 213-594). A 15-20 cm bowel segment was harvested, and included sigmoid (2) and ileum (4). 5 (83\%) patients required the ICU postoperatively. Median length of stay was 25 days (range 1157). $4(67 \%)$ patients had major postoperative complications.

Follow-up: All patients are dry with no urethral leakage at a median of 41.4 months (range 4-67). Renal function is stable or improved in all patients.

Conclusions Incontinent urinary diversion should be considered in young adult patients with SB who are not capable of continuing CIC. These patients are highly complex with significant comorbidities and risk of sub- stantial surgical complications. Clear expectations and goals should be established preoperatively.

Dextranomer Endoscopic Injections for the Treatment of Neuropathic Urinary Incontinence: Evaluation of the Supra Pubic Approach

Lottmann, Henri ${ }^{1}$, Alova, Ilona ${ }^{1,2}$

${ }^{1}$ Hôpital Necker-enfants malades

${ }^{2}$ Ellen Poidatz Foundation

Background We previously reported a 52\% success rate using trans urethral dextranomer endoscopic injections for neuropathic incontinence in 46 patients (dry $40 \%$, significantly improved $12 \%)^{1}$. Results were significantly better in females (68\%) than in males $(38 \%)$. Up to $80 \%$ success were reported by others ${ }^{2}$.

Methods Since 2010, 24 patients (5-18 years) with mainly congenital (22) neuropathic bladder with sphincteric incompetence have been enrolled.

Preoperative evaluation consisted in medical history, incontinence charts, urine culture, urinary tract ultrasound and videourodynamics. This evaluation was repeated at six months and one year after treatment and then on a yearly basis, except for videourodynamics performed only when necessary for further management. Thirteen patients had one six two and 3 three treatment sessions. Mean injected volume was $6 \mathrm{ml}$ per session. At each evaluation, the patient was classified as cured (dryness interval of four hours), significantly improved (minimal incontinence requiring no more than one pad per day; no further treatment required) both defining treatment success, and treatment failure when no significant improvement was observed. Follow-up ranged from 6 to 72 months.

Results There were no serious adverse events or complications observed. 5/24 (20\%) patient are dry, 7/24 $(30 \%)$ are significantly improved, and $12 / 24(50 \%)$ patients had treatment failure. Female patients achieved a higher success rate $(60 \%)$ than males $(43 \%)$; otherwise there were no significant clinical or urodynamical criteria predictive of success or failure.

Conclusions Endoscopic treatment of neuropathic urinary sphincter insufficiency with Dextranomer based implants, is effective in $50 \%$ of patients, particularly females. In our hands the supra pubic is not superior to the trans urethral approach with less totally dry patients 1-J Urol, 2012, 188,5: 1905 2-J Urol 2010; 184: 1644. 
Urologic Outcomes in Spina Bifida Patients Undergoing Transitional Care

Tan, Jubilee ${ }^{2}$, Hopson, Betsy D. ${ }^{1}$, Li, Peng ${ }^{2}$, Lloyd, L. Keith ${ }^{2}$, Joseph, David B. ${ }^{1}$, Dangle, Pankaj P. ${ }^{1}$, Wilson, Tracey S. ${ }^{2}$

${ }^{1}$ UAB-Children's of Alabama

${ }^{2} U A B$

Background Young adults with Spina Bifida (SB) are at high risk for urologic comorbidities and surgical intervention. Efforts have been made to transition patients from pediatric to adult care with the goal of preventing deterioration of renal function and improving quality of life outcomes. The objective of this study was to determine if enrollment in a transitional clinic resulted in improved urologic outcomes.

Methods A retrospective chart review was conducted of patients in the Adult Multidisciplinary SB Clinic at UAB from Oct 2010 to Oct 2016.

$38 \%$ of these patients transitioned from pediatric to adult care. Age at first adult clinic visit, adherence to follow up, surveillance upper tract imaging, type of bladder management, use of GU medications, and urinary continence were assessed. Prevalence of urologic sequelae was also noted, including: hydronephrosis, vesicoureteral reflux, renal scarring, hypertension, recurrent urinary tract infections, CKD or ESRD, and urolithiasis. Surgical procedures performed in adulthood were also recorded.

Results One hundred eighty-seven patients were evaluated, 71 were transitioned (TR) and 116 were not transitioned (NTR). TR patients were younger than NTR patients $(24.1$ vs $35.9, p<0.001)$ at the time of the study. $70.4 \%$ of patients in the TR group had their initial evaluation before 21 years old compared to $23.3 \%$ in the NTR group $(p<0.0001)$. Methods for bladder management were significantly different between the two groups $(P=0.0019) .78 .9 \%$ of patients in the TR group performed CIC compared to $57.8 \%$ in the NTR group. Patients in the TR group were less likely to undergo urologic procedures as an adult.

Conclusions Transition of care may significantly affect urologic outcomes, but more long-term data is required. This study suggests that as patients with SB reach adulthood, the absence of routine urologic care could increase their need for urologic surgeries and could negatively impact their overall bladder management.
Pregnancy in Spina Bifida: Implications for Transitional Care

Shepard, Courtney L., Yan, Phyllis, Hollingsworth, John M., Kraft, Kate H.

University of Michigan

Background Sexual functioning and fertility is a major concern for adolescent and young adult patients with Spina Bifida but is not routinely addressed by providers. As genitourinary specialists who typically follow these patients from birth to young adulthood, pediatric urologists typically counsel patients on this topic. For adequate counseling, pediatric urologists should have an understanding of obstetrical outcomes for women with Spina Bifida (SBW). Current knowledge regarding pregnancy in SBW is limited. We evaluate patterns of delivery in SBW compared to women without Spina Bifida (NSB).

Methods Using the National Inpatient Sample, a 20\% stratified sample of all patient discharges, we identified women hospitalized for a delivery in 2003-2013. We performed temporal trends analysis and analyzed differences in characteristics between SBW and NSB utilizing parametirc and nonparametric bivariant statistics.

Results We identified 10,147 hospitalizations for delivery amongst SBW and 42,197,763 amongst NSB. Of all hospitalizations for deliveries, the percent of deliveries by SBW is significantly increasing $(p<0.001)$. SBW hospitalized for a delivery differed from NSB hospitalized for a delivery in that they had a higher number of comorbidies and were more likely to be white, have medicare or private insurance, live outside a city, deliver at an urban teaching hospital, and undergo a caesarean section $(52.4 \%$ of SBW versus $31.9 \%$ of NSB) (all $p<0.001$ ). They did not differ significantly by age or socioeconomic status. Of all hospitalizations for delivery amongst SBW, $26 \%$ occur by age 22 .

Conclusions There are significant differences in the characteristics and mode of delivery between SBW and NSB hospitalized for a delivery. Many deliveries occur during the transitional period. Pediatric urologists should intentionally discuss sexual activity with SBW and offer gynecology referral to review appropriate screening and family planning options. 
Incidence and Management of Pelvic Organ Prolapse in Adult Spina Bifida Patients

Kielb, Stephanie, Liu, Joceline, Vo, Amanda, Hamoui, Nabeel, Lewicky-Gaupp, Christina

Northwestern University Feinberg School of Medicine

Background Pelvic organ prolapse (POP) affects $40 \%$ of women, and increases with parity and age. Failure to treat POP may lead to incomplete bladder or bowel emptying, ulcerations, and ureteral obstruction with hydronephrosis. Although the level of spinal cord lesion is heterogeneous, the majority of SB patients are affected at the thoracic or lumbar spine. Support of organs by the pelvic floor, innervated by the pudendal nerve and sacral nerve roots S3-S5, is likely affected in SB patients; yet there is no research on incidence or management of POP in these patients. We report our experience with POP in adult female SB patients at a tertiary care urology clinic.

Methods Retrospective review of SB patients $\geqslant 18 \mathrm{yrs}$ with a documented POP quantification exam between 2006 and 2014. Demographics, gestation, parity, POP quantification and prolapse symptoms were obtained.

Results Thirty-three SB patients were identified with a mean age of 33.2 years. Five $(15.2 \%)$ had stage 0 prolapse, $12(36.4 \%)$ stage $1,12(36.4 \%)$ stage $2,3(9.1 \%)$ stage 3 , and $1(3.0 \%)$ stage 4 . Of the 16 patients with advanced POP (stage 2 or greater), only $6(37.5 \%)$ reported POP symptoms. All 6 symptomatic patients endorsed sensation of a vaginal bulge. Two of the 6 patients also reported dyspareunia and 1 patient with advanced POP presented with vaginal bulge, noted by a caregiver, and cervical bleeding. Twenty-four $(72.7 \%)$ were nulliparous, and 12/24 nulliparous patients (50\%) demonstrated prolapse. Two patients underwent vaginal hysterectomy with uterosacral suspension and two were managed with partial cervicectomy.

Conclusions Patients with SB are more likely to have POP than the general population and the majority of patients with prolapse are asymptomatic. Assessment of pelvic organ prolapse should be included in the evaluation of adult SB females due to the low rate of symptoms and potential impact on both urinary and bowel function.

\section{Sexual Activity and Function of Adult Men with Spina Bifida}

Szymanski, Konrad $^{1}$, Hensel, Devon ${ }^{2}$, Wiener, John $\mathrm{S}^{3}$, Whittam, Benjamin ${ }^{1}$, Kaefer, Martin ${ }^{1}$, Chan, Katherine $^{1}$, Rink, Richard ${ }^{1}$, Cain, Mark ${ }^{1}$, Misseri, Rosalia $^{1}$

\author{
${ }^{1}$ Riley Hospital for Children \\ ${ }^{2}$ Department of Pediatrics and Department of Sociol- \\ ogy, Indiana University Purdue University Indianapo- \\ lis \\ ${ }^{3}$ Section of Pediatric Urology, Duke University Medi- \\ cal Center
}

Background Little is known about sexual function of men with Spina Bifida (SB). The aim of this study was to assess sexual activity and erectile dysfunction (ED) in men with SB.

Methods We performed an international online survey of men $\geqslant 18$ yo with SB. Participants were recruited via $\mathrm{SB}$ clinics and social media. We collected data on demographics, ambulation (Hoffer classification), penile rigidity (Erection Hardness Score), sexual activity and ED (International Index of Erectile Function). Non-parametric tests were used for analysis.

Results Of 69 men (median age 35, 62.3\% shunted, $27.1 \%$ community ambulators), $50.7 \%$ were single (24.6\% in a relationship, $18.8 \%$ married). Overall, $35.3 \%$ reported full penile rigidity with erections, more commonly in men with better ambulation $(p=0.04)$. $66.2 \%$ of men achieved orgasm and $78.3 \%$ ejaculated. Lifetime solo masturbation was reported by $91.3 \%$, partnered non-genital contact: $85.5 \%$, mutual masturbation: $78.3 \%$, and partnered intercourse: $75.4 \%$ (62.3\% vaginal). Median age at first sexual contact was 19 years, median 3.5 lifetime partners. Of 25 men who previously used phosphodiasterase type 5 (PDE5) inhibitors, $76.0 \%$ had improved erections and $56.0 \%$ improved intercourse.

Overall, $46.3 \%$ of men reported non-genital erogenous zones as most pleasurable, more commonly in men with lower ambulation $(p=0.03)$. Nipples $(38.7 \%)$ and chest $(22.6 \%)$ were most common. Among 18 men $(26.9 \%)$ who attempted sexual intercourse in the last 4 weeks, $61.1 \%$ had ED (16.7\% severe, $5.6 \%$ moderate, $22.2 \%$ mild-moderate, $22.2 \%$ mild). In this group, ED was present in $7 / 7$ men with less than full penile rigidity, compared to $4 / 11(36.4 \%)$ with full penile rigidity $(p=0.01)$.

Conclusions While ED is common in men with SB, PDE5 inhibitors are commonly used and may benefit this population. Higher ambulatory status portends better erectile function. Partnered sexual activity is common, although may not involve penetrative intercourse. 